\title{
Correspondence between rats and humans in the utilization of retrospective and prospective codes
}

\author{
RAYMOND P. KESNER and MONIQUE J. DESPAIN \\ University of Utah, Salt Lake City, Utah
}

\begin{abstract}
Rats were presented with $2,4,6,8$, or 10 spatial locations on a 12 -arm radial maze and on a subsequent test were required to choose between a place previously visited and a novel place. The animals were reinforced for entering the novel spatial location. During learning, animals showed an increase in errors as the number of places to be remembered increased from 2 to 8 , indicating the use of a retrospective memory code. These animals also showed a decrease in errors as the number of places to remember increased from 8 to 10 , indicating the use of a prospective memory code. In an analogous mnemonic task, college students were presented with 2,4 , $6,8,10,12$, or $14 \mathrm{Xs}$, shown one at a time, marked on a grid of 16 squares. On a subsequent test, the college students were asked to choose between a novel $X$ and one that had appeared previously. Subjects were asked to circle the novel X. On the basis of their verbal reports, the subjects were divided into two groups reflecting different memory coding strategies. The subjects who reported a retrospective coding strategy showed an increase in errors as the list length increased. The subjects who reported a retrospective and prospective strategy showed an initial increase in errors for list lengths of 2 to 8 items followed by a decrease in errors for list lengths of 8 to 14 items. The results are interpreted to reflect a correspondence between animals and humans in the use of coding strategies aimed at facilitating memory performance for long lists of information.
\end{abstract}

Based on the assumption of evolutionary continuity of mental processes between animals and humans, there has been an ever-increasing interest in studying parallels between animals and humans in terms of cognitive functions. This has led to the finding of a variety of comparable mnemonic functions in animals and humans. For example, animals display serial-position curves for lists of items (DiMattia \& Kesner, 1984; Kesner \& Novak, 1982; Wright, Santiago, Sands, Kendrick, \& Cook, 1985), utilize rehearsal and exhaustive scanning processes within short-term memory (Ellis, Clegg, \& Kesner, 1984; Kendrick \& Rilling, 1984), learn lists of paired associates (Petrides, 1985), and learn about spatial environments requiring use of cognitive maps (DiMattia \& Kesner, in press; Gould, 1986; Menzel, 1978; Morris, 1981). It was shown recently that rats can use retrospective and prospective memory codes when required to remember items within long lists (Cook, Brown, \& Riley, 1985). More specifically, in Cook et al.'s study a rat was presented on any one trial with $2,4,6,8$, or 10 items (places) on a 12-arm radial arm maze, followed 15 min later by a win-shift test consisting of a choice between a place previously visited and a novel place. The animal was reinforced for entering the novel spatial location. During learning, the animals showed an increase in errors as the number of places to be remembered increased from 2 to 6 , reflecting the use of a retrospective memory code. These animals

Support for this research was provided by NIH Grant ROINS2077103. Requests for reprints should be sent to Raymond P. Kesner, Department of Psychology, University of Utah, Salt Lake City, UT 84112. also showed a decrease in errors as the number of places to be remembered increased from 6 to 10 , reflecting the use of a prospective memory code.

It should be noted, however, that inferences concerning the use of retrospective and prospective codes may be problematic, especially because of the inability to assess the utilization of these codes by means other than verbal report. It is, therefore, of paramount importance to develop analogous tasks for humans in order to make comparisons with animals. Furthermore, since the emphasis in memory research has been on the use of retrospective codes, it would be of real interest to test to what degree humans utilize prospective coding strategies in order to remember long lists of events (in this case spatial locations). Thus, the purposes of the present study were to replicate Cook et al.'s (1985) study with rats and to test humans using a comparable set of procedures and spatial location information.

\section{METHOD}

\section{Subjects: Animals}

Ten male Long-Evans rats, aged 4 to 6 months at the start of the experiment, were used. Each animal was individually housed and was maintained at approximately $80 \%-85 \%$ of its free-feeding weight. Animals had free access to water. The animal cages were kept in a laboratory with a 12-h light-dark cycle.

\section{Apparatus}

The apparatus was an elevated $(80 \mathrm{~cm}$ from the floor) 12 -arm radial maze, painted white. The maze consisted of a center platform $40 \mathrm{~cm}$ in diameter with 12 arms $(9 \mathrm{~cm}$ in width, and $75 \mathrm{~cm}$ in length) radiating from the platform. $A$ transparent $k$-in. Plexi- 
glas guillotine door was set at the entrance to each arm to control the animals' access to the arm. By means of a series of pulleys and strings, each door could be opened and closed from an adjacent lab room. At the distal end of each arm, a small hole $(2.5 \mathrm{~cm}$ in diameter and $1.5 \mathrm{~cm}$ in depth) was drilled to serve as a food well. The maze was located in a room with a wide variety of extramaze cues, including a set of pictures on the wall and a small black desk near the maze.

\section{Training}

Initially, each animal was allowed to explore the maze on a daily basis and to eat food scattered around the maze for $10 \mathrm{~min}$ per day. This procedure was followed for 5 to 6 days. The animals were then trained using Olton and Samuelson's (1976) procedure. In this phase, the animals were placed on the center platform of the maze and each arm was baited with a piece of Froot Loops breakfast cereal (Kellogg Co.) in the food well. The animals were allowed to visit all arms to get food for a 20 -min session. This training was continued daily until the animals obtained food from all arms within 20 choices or within a $20-$ min time period. All animals received one session per day for 15 days.

After reaching criterion, the animals were tested using the procedures described by Cook et al. (1985, Experiment 4). Each training trial consisted of a study phase and a test phase. During the study phase a sequence of $2,4,6,8$, or 10 arms was presented, one arm at a time. The arms were randomly selected, with the constraint that no 3 adjacent arms be presented in one sequence.

Within a 5-day period, each sequence and point of interpolation (POI), or list length, was randomly selected without replacement. At the end of each sequence of visits to appropriate arms and consumption of $1 / 4$ piece of Froot Loop reinforcement from each arm, the animal was removed from the maze and returned to its home cage.

Fifteen minutes later the animal was placed in the center platform to commence the test phase. During the test phase, two adjacent doors were opened-one that had been visited during the study phase and one that had not. The correct response was to select the novel arm; that is, the animal had to use a win-shift strategy. On $50 \%$ of the trials, the correct response was the door on the right, and on the other $50 \%$ the correct response was the door on the left. A correct response was rewarded with a whole piece of Froot Loop cereal. Immediately after making a choice, the animal was given a second test. Two adjacent doors that had not been used on the first test were opened-one that had been visited during the study phase and one that had not. Furthermore, on a random basis, the choices on one test were selected from spatial locations visited during the first half of the list, and the choices on the other test were selected from locations visited during the second half of the list. This procedure was used in order to minimize possible confounding effects from the serial position of items within each list. A correct response was again based on a win-shift strategy. Only one training trial (consisting of a study phase and a test phase [two tests]) was given per day. Each animal was given a total of 40 tests, with 8 tests of each POI $(2,4,6,8$, or 10$)$.

\section{Subjects: Humans}

Twenty-four students from a University of Utah introductory psychology class were tested in groups of 8 . Each trial was divided into a study phase and a test phase. During the study phase, the subjects were presented with $2,4,6,8,10,12$, or $14 \mathrm{Xs}$, shown one at a time. Each $X$ was printed in a specific location on a $20.2 \times 27.9 \mathrm{~cm}$ transparency that contained a grid of 16 squares. Only one $X$ appeared at a time for a 5-sec duration. The stimuli were presented on a screen with an overhead projector.

The subjects received 28 trials, 4 trials of each list length, with an intertrial interval of $10 \mathrm{sec}$. For each trial both the locations of each $\mathrm{X}$ and the list length were randomly arranged.
Five seconds after the study phase, the subjects were given two tests in which they were asked to choose between an X that had not appeared in the study phase (novel) and one that had appeared in the study phase. The subjects were asked to circle the novel X; that is, they had to use a win-shift rule. The tests containing the Xs were presented to the subjects on individual stacks of paper.

After all the tests had been completed, the subjects were presented with a questionnaire containing two questions: (1) Did you use different codes for remembering short versus long lists? (2) What strategy did you use? These questions were designed to aid in categorizing the strategies used to remember the various list lengths.

\section{RESULTS}

\section{Animals}

Behavioral performance (mean number of errors) is shown in Figure 1 as a function of POI. The figure shows that the rats displayed an increase in the number of errors up to a set size of 8 items, followed by a decrease in the number of errors for a set size of 10 items. Two of the 10 animals showed an increase, rather than a decrease, in number of errors for a set size of 10 items. It should be noted that, based on eight observations, four errors represent chance performance, whereas two or fewer errors represent significantly better than chance performance.

A one-way analysis of variance on the mean number of errors as a function of POI revealed a significant effect of POI $[F(4,36)=4.5, p<.01]$. Subsequent Duncan range tests revealed that there was a significant increase in errors for a POI of 8 items compared with a POI of $2,4,6$, or 10 items $(p<.05)$.

\section{Humans}

The human subjects were divided into two groups on the basis of their written responses to the two questions concerning the strategy they used to remember the various list lengths. Comments that were judged to be in the retrospective category included, for example, "I remembered by drawing pictures of shapes in my head with lines by the pattern of the Xs" and "I tried to form patterns with the filled-in squares." Half of the students used a

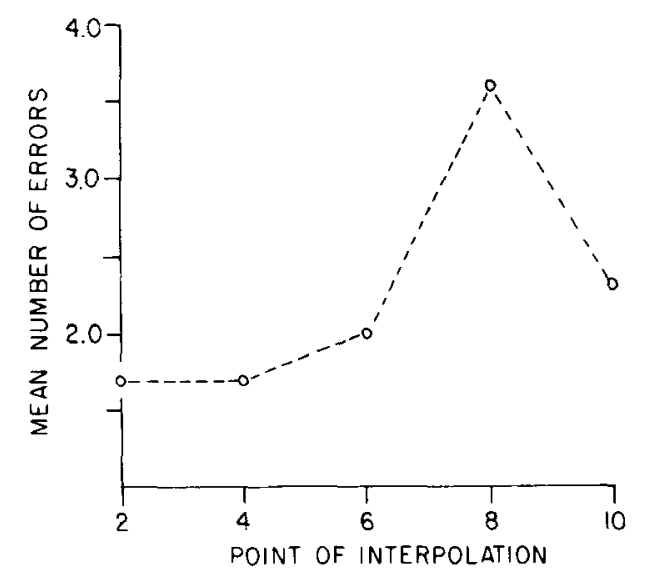

Figure 1. Mean number of errors as a function of point of interpolation (list length). 
retrospective strategy; that is, they tried to remember all the items that were presented in the study phase. The other half of the students used both retrospective and prospective strategies; that is, they shifted their strategy to try to remember the remaining items when the list lengths were long. Comments that were judged to be in the retrospective/prospective category included, for example, "I formed a positive geometric shape. When more than $6 \mathrm{Xs}$ were shown I started a negative geometric shape" and "When there were more spaces I remembered Xs, when there were more Xs I remembered spaces."

Because the error rate was rather low, it was decided to depict the results using the total number of errors rather than the average number of errors. The results, shown in Figure 2, indicate that the students who used a retrospective code showed an increase in errors with an increase in POI. In contrast, the students who used a retrospective/prospective code increased in errors up to a POI of 8 items and then showed a decrease in errors with longer list lengths.

A two-way analysis of variance on the total number of errors as a function of POI for the subjects who used a retrospective code and those who used a retrospective/prospective code revealed a significant group effect $[F(1,22)=6.9, p<.05]$ and a significant POI effect $[F(6,132)=6.0, p<.01]$, as well as a significant group $\times$ POI interaction $[F(6,123)=2.8, p<.05]$. Subsequent Duncan range tests revealed that for the retrospective code group there were significantly more errors at the longest POI (14 items) than at POIs of 2, 4, 6, $(p<.01)$, or $12(p<.05)$ items. There were also more errors at POIs of 8 or 10 items than at a POI of 2 items $(p<.05)$.

Subsequent Duncan range tests for the retrospective/ prospective code group revealed that there were signifi-

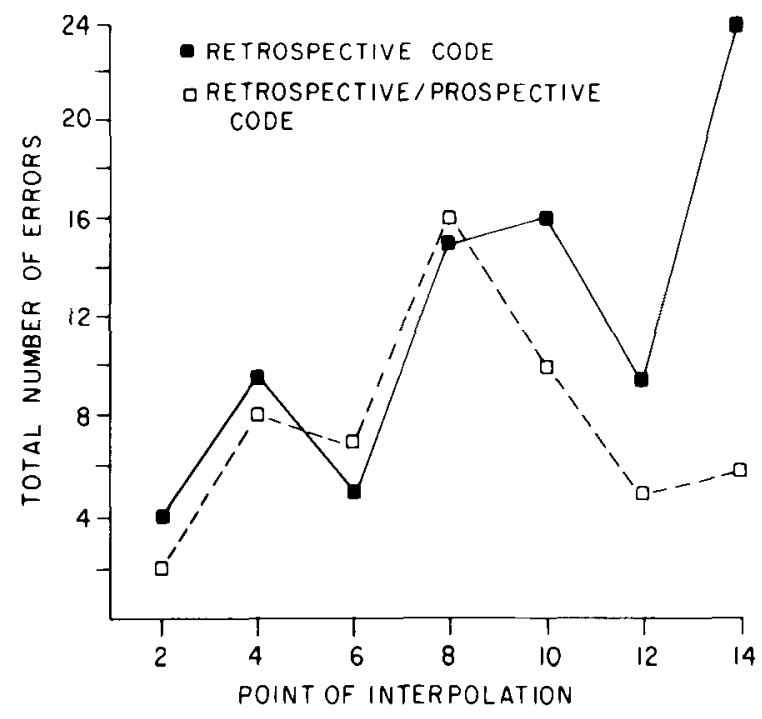

Figure 2. Total number of errors as a function of point of interpolation (list length) for subjects using retrospective and retrospective/prospective codes. cantly more errors at a POI of 8 items than at a POI of $2(p<.01)$ or 12 or 14 items $(p<.05)$.

\section{DISCUSSION}

The present study indicates that college students use at least two types of strategies in coding information within long lists. The first strategy is to remember all of the previously presented items; that is a retrospective strategy, which should result in increased errors as the list length increases. Indeed, the largest number of errors occurred for the longest list length. The second strategy is to shift somewhere in the middle of the list from a strategy of remembering all of the previously presented items to remembering the items that still remain in the list, that is, a retrospective/prospective strategy. This strategy should result in an initial increase in errors, followed by a decrease as list length increases. Indeed, the largest number of errors occurred in the middle of the list. The latter error pattern was seen in Cook et al.'s (1985) study with rats and in the present study when the list length increased. The observation that both humans and animals use a prospective code in remembering long lists of items provides an opportunity to analyze the neural basis of prospective coding of information in animals and humans. A recent series of studies showed that rats with parietal (Kametani $\&$ Kesner, in press) or frontal (Kesner, in press) lesions do not show the ability to use a prospective code, whereas rats with hippocampal lesions are impaired in using retrospective and prospective codes (Kametani \& Kesner, in press).

Even though, in the present study, there were a few differences between the procedure used with rats and that used with humans (the use of spaced vs. massed trials, longer set size for the human subjects, a short vs. long delay between study phase and test phase, and use of a different type of reinforcement), there were a sufficient number of procedural similarities (including the use of spatial location information and two tests for each study phase) to permit a comparison between rats and humans. Thus, the similarity in the pattern of results suggests the possibility that rats indeed utilize both retrospective and prospective codes in remembering long lists of information. This explanation could account for the excellent ability of rats to remember all spatial locations in a 17-arm maze (Olton, Collison, \& Werz, 1977).

In conclusion, the present study provides yet another demonstration that comparable mnemonic functions can be found in animals and humans.

\section{REFERENCES}

Cook, R. G., Brown, M. G., Riley, D. A. (1985). Flexible memory processing by rats: Use of prospective and retrospective information in the radial maze. Journal of Experimental Psychology: Animal Behavior Processes, 11, 453-469.

DiMattia, B. V., Kesner, R. P. (1984). Serial position curves in rats: Automatic vs. effortful information processing. Journal of $E x$ perimental Psychology: Animal Behavior Processes, 10, 557-563. 
DiMattia, B. V., \& Kesner, R. P. (in press). Spatial cognitive maps: Differential role of parietal cortex and hippocampal formation. $B e^{-}$ havioral Neuroscience.

Elus, M. E., CleGG, D. K., Kesner, R. P. (1984). Exhaustive memory scanning in Rattus norvegicus: Recognition for food items. Journal of Comparative Psychology, 98, 194-200.

GouLD, J. L. (1986). The locale map of honey bees: Do insects have cognitive maps? Science, 232, 861-863.

KametaNi, H., \& KeSner, R. P. (in press). Retrospective and prospective coding of information: Dissociation of parietal cortex and hippocampal formation. Behavioral Neuroscience.

KendricK, D. F., Rilung, M. (1984). The role of interpolated stimuli in the retroactive interference of pigeon short-term memory. Animal Learning \& Behavior, 12, 391-401.

KESNER, R. P. (in press). Retrospective and prospective coding of information: Role of the medial prefrontal cortex. Experimental Brain Research.

Kesner, R. P., Novak, J. M. (1982). Serial position curve in rats: Role of the dorsal hippocampus. Science, 218, 173-175.

MENZEL, E. W. (1978). Cognitive mapping in chimpanzees. In S. H.
Hulse, H. Fowler, \& W. K. Honig (Eds.), Cognitive aspects of animal behavior. Hillsdale, NJ: Erlbaum.

MorRIs, R. G. M. (1981). Spatial localization does not require the presence of local cues. Learning \& Motivation, 12, 239-260.

Olton, D. S., Colluson, C., \& Werz, M. A. (1977). Spatial memory and radial arm maze performance of rats. Learning \& Motivation, 8, 289-314.

Olton, D. S., SAmUELSON, R. J. (1976). Remembrance of places passed: Spatial memory in rats. Joumal of Experimental Psychology: Animal Behavior Processes, 2, 97-116.

PETRIDEs, M. (1985). Deficits in nonspatial conditional associative learning after periarcuate lesions in the monkey. Behavioral Brain Research, 16, 95-101.

Wright, A. A., Santiago, H. C., Sands, S. F., Kendrick, D. F., \& Cook, R. G. (1985). Memory processing of serial lists by pigeons, monkeys, and people. Science, 229, 287-289.

(Manuscript received October 20, 1987; revision accepted for publication January 28, 1988.) 